\title{
Baryons, monopoles and dualities in Chern-Simons-matter theories
}

\author{
Ofer Aharony \\ Department of Particle Physics and Astrophysics, Weizmann Institute of Science, \\ Rehovot, 7610001 Israel \\ E-mail: Ofer.Aharony@weizmann.ac.il
}

ABSTRACT: There is significant evidence for a duality between (non-supersymmetric) $\mathrm{U}(N)$ Chern-Simons theories at level $k$ coupled to fermions, and $\mathrm{U}(k)$ Chern-Simons theories at level $N$ coupled to scalars. Most of the evidence comes from the large $N$ 't Hooft limit, where many details of the duality (such as whether the gauge group is $\mathrm{U}(N)$ or $\mathrm{SU}(N)$, the precise level of the $\mathrm{U}(1)$ factor, and order one shifts in the level) are not important. The main evidence for the validity of the duality at finite $N$ comes from adding masses and flowing to pure Chern-Simons theories related by level-rank duality, and from flowing to the non-supersymmetric duality from supersymmetric dualities, whose finite $N$ validity is wellestablished. In this note we clarify the implications of these flows for the precise form of the duality; in particular we argue that in its simplest form the duality maps $\mathrm{SU}(N)$ theories to $\mathrm{U}(k)$ theories, though there is also another version relating $\mathrm{U}(N)$ to $\mathrm{U}(k)$. This precise form strongly affects the mapping under the duality of baryon and monopole operators, and we show, following arguments by Radičević, that their mapping is consistent with our claims. We also discuss the implications of our results for the additional duality between these Chern-Simons matter theories and (the UV completion of) high-spin gravity theories on $A d S_{4}$. The latter theories should contain heavy particles carrying electric and/or magnetic charges under their $\mathrm{U}(1)$ gauge symmetry.

KeYwords: Duality in Gauge Field Theories, Chern-Simons Theories, 1/N Expansion

ArXiv EPrint: 1512.00161 


\section{Contents}

1 Introduction 1

2 Dualities between Chern-Simons theories 3

3 Baryons and monopoles in Chern-Simons-matter theories $\quad 6$

4 Mapping of baryons and monopoles under the duality 4

$\begin{array}{lll}5 & \text { Mapping to high-spin gravities } & 10\end{array}$

\section{Introduction}

One of the main tools to study strongly coupled field theories is duality - the claim that certain strongly coupled theories are equivalent to (generally different) weakly coupled theories. This phenomenon was first discovered in two space-time dimensions, and in the past twenty years many examples of duality were discovered in supersymmetric theories in higher dimensions. However, the theories which we would most like to understand at strong coupling are non-supersymmetric theories in three and four space-time dimensions. For such theories there is only one conjectured duality so far — the duality [1-3] between three dimensional $\mathrm{U}(N)$ Chern-Simons (CS) theories coupled to fermions, and three dimensional $\mathrm{U}(\tilde{N})$ Chern-Simons theories coupled to scalars (there is a similar duality also for $\mathrm{SO}(N)$ theories, that we will not discuss here).

This non-supersymmetric duality was originally motivated by the duality $[4,5]$ of both types of theories, in the large $N$ limit, to high-spin gravity theories on $A d S_{4}$ constructed by Vasiliev [6]. It was then corroborated by many exact computations in the large $N$ limit (with fixed $N / k$, where $k$ is the Chern-Simons level), which are all consistent with the duality, and by the classification of theories with slightly broken high-spin symmetries $[7,8]$. It was also generalized $[9,10]$ to theories with additional fields, including supersymmetric field theories for which some of the dualities that arise were already previously known [11-14].

All known dualities that are valid at large $N$ also have a generalization to finite $N$, so it is natural to conjecture that this is true also here. However, it is difficult to test this conjecture, since it is not known how to perform the large $N$ computations at finite $N$ (and even the $1 / N$ corrections have not been computed yet). In particular, the large $N$ tests do not distinguish between theories whose level is shifted by a finite number in the large $N$ limit, nor between $\mathrm{U}(N)$ and $\mathrm{SU}(N)$ theories. So far there are just two pieces of evidence for the finite $N$ duality. One piece of evidence comes from deforming the theories related by the duality by a fermion mass term [3]. At low energies the CS-fermion theory flows to a 
pure Chern-Simons theory, with a level depending on the sign of the mass term. Similarly, the CS-scalar theory flows to a pure Chern-Simons theory, with the rank depending on the sign of the mass term. The resulting Chern-Simons theories must then be equivalent, and indeed, for a specific choice of the order one shift in the level, the theories (for both signs of the mass) are related by level-rank duality [15-19]. This flow can be used to fix the finite shift in the Chern-Simons level.

The second piece of evidence is that one can flow from supersymmetric dualities, for which there is a lot of evidence at finite $N$, to the non-supersymmetric dualities $[10,20]$. This flow is under precise control at large $N$, but the same flow works also at finite $N$, at least as long as the finite- $N$ corrections do not lead to qualitative changes in the flow (such as new operators becoming relevant).

In principle, both methods should enable us to determine whether the duality relates $\mathrm{SU}(N)$ theories or $\mathrm{U}(N)$ theories, and also to determine the precise level of the $\mathrm{U}(1)$ factor in $\mathrm{U}(N)$ (which also does not affect the large $N$ tests). However, this was not done in the previous literature on the subject. In section 2 we use this method to fix the precise form of the duality. We find that there is one version of the duality which relates $\mathrm{SU}(N)$ to $\mathrm{U}(\tilde{N})$ theories, and another that relates $\mathrm{U}(N)$ to $\mathrm{U}(\tilde{N})$ theories, but where the level of the $\mathrm{U}(1)$ is not equal to the level of $\mathrm{SU}(N)$.

The subtle distinctions above do not affect much of the physics of these theories, but it greatly modifies the form of operators whose dimension scales as a power of $N$ in the large $N$ limit. $\mathrm{SU}(N)$ CS-matter theories contain baryon operators charged under a $\mathrm{U}(1)$ global symmetry, while $\mathrm{U}(N)$ theories do not. $\mathrm{U}(N)$ theories contain monopole operators charged under a U(1) global symmetry, and the form and dimension of the gauge-invariant globally-charged monopole operators depends strongly on the level of the U(1) group (and not just the $\mathrm{SU}(N)$ group). Thus, we can learn about the precise form of the duality by matching these "non-perturbative" (in the sense of the $1 / N$ expansion) operators between the two sides.

A naive computation of the monopole dimensions on the two sides of the duality of [1-3] leads to a serious mismatch, apparently contradicting the duality [21, 22]. It was suggested in $[23]$ that this mismatch could be partly resolved if the duality relates $\mathrm{SU}(N)$ theories to $\mathrm{U}(\tilde{N})$ theories. Our discussion, motivated by [23], shows that this is indeed the case, and we will show that this actually completely resolves the mismatch between the two sides.

In section 3 we review the properties of the baryon and monopole operators, in CSfermion and CS-scalar theories. In some cases the dimensions of these operators in the large $N$ 't Hooft limit are known, and in other cases they are only known at weak coupling. Some of these operator dimensions turn out to scale as $N$ in the 't Hooft limit, and others as $N^{3 / 2}$.

In section 4 we show that the baryon and monopole operators precisely map to each other under the duality in the $\mathrm{SU}(N) \leftrightarrow \mathrm{U}(\tilde{N})$ case, and that the monopole operators precisely map to each other in the $\mathrm{U}(N) \leftrightarrow \mathrm{U}(\tilde{N})$ case. More precisely, we cannot really show this since we do not know how to compute the dimensions at all values of the coupling. But we find that the quantum numbers of these operators are the same on both sides, and that the weak coupling limit of the dimension in one theory maps precisely to the weak 
coupling limit in the other theory, suggesting that the large $N$ value of this dimension is not corrected as a function of the 't Hooft coupling. This was shown for a specific operator (the monopole in CS-fermion theories) in [23], but the duality suggests that it should be correct also for the other operators.

In section 5 we discuss how these non-perturbative operators should appear in (the UV completion of) the high-spin gravity theories on $A d S_{4}$ that are dual to these ChernSimons matter theories. These theories have a $\mathrm{U}(1)$ gauge field on $A d S_{4}$ that couples to the baryon number or to the monopole number; we argue that these two charges are related by electric-magnetic duality. We argue that the high-spin gravity theories should contain (in some $\operatorname{SL}(2, Z)$ frame of the electric-magnetic duality of this $\mathrm{U}(1)$ gauge field) magnetically-charged particles with an energy scaling as $N$ (so that they could be classical solutions of the Vasiliev action), and electrically-charged particles with an energy scaling as $N^{3 / 2}$. The energy of these particles (in Planck units) should depend strongly on the theta angle appearing in the Vasiliev equations of motion. It would be interesting to understand the implications of these particles for the non-perturbative completion of the high-spin gravity theories.

\section{Dualities between Chern-Simons theories}

In this section we review the form of level-rank dualities between Chern-Simons theories, and clarify their relation to dualities between Chern-Simons-matter theories. We discuss here only $\mathrm{SU}(N)$ and $\mathrm{U}(N)$ theories.

The value of the Chern-Simons level $k$ of an $\mathrm{SU}(N)$ gauge theory depends (as many coupling constants do) on the regularization procedure. There are two common regularizations that are used for Chern-Simons(-matter) theories. One involves starting from a Yang-Mills-Chern-Simons theory at high energies, and flowing to low energies where the Yang-Mills term becomes irrelevant. In most diagrams gluon loops are cut off by the fact that the $3 d$ Yang-Mills theory is super-renormalizable. In this regularization, $k$ is quantized (we normalize it so that it is quantized to be an integer in the absence of matter fields), and can take any integer value.

Another common regularization of Chern-Simons(-matter) theories is dimensional regularization. This is somewhat subtle since the Chern-Simons interaction involves an epsilon-symbol, but there is a standard way to perform it [24]. The level (coupling constant) $\hat{k}$ appearing in this regularization is shifted compared to the previous one, such that for any $k \neq 0$,

$$
\hat{k}=k+N \operatorname{sign}(k) .
$$

In particular, $|\hat{k}|>N$. This shift arises due to a one-loop diagram with a gluon loop. It is not present for $\mathrm{U}(1)$ gauge groups.

When discussing dualities, it is important to be consistent about the regularization. The Chern-Simons level-rank duality is usually discussed in the Yang-Mills regularization. When we have a $\mathrm{U}(N)=(\mathrm{SU}(N) \times \mathrm{U}(1)) / Z_{N}$ theory, we can apriori have different levels for $\mathrm{SU}(N)$ and $\mathrm{U}(1)$ (consistent with the global structure of the gauge group). We will 
denote $\mathrm{U}(N)$ CS theories as $\mathrm{U}(N)_{k_{1}, k_{2}}$ where $k_{1}$ is the $\mathrm{SU}(N)$ level (quantized to be an integer), and $k_{2}$ is the $\mathrm{U}(1)$ level (normalized so that $\mathrm{U}(N)_{k, k}$ is the theory one gets by a Chern-Simons term involving a trace in the fundamental representation of $\mathrm{U}(N)$; note that other normalizations are often used in the literature). In this convention, Chern-Simons theories are invariant under a level-rank duality relating ${ }^{1}[15-19]$

$$
\mathrm{SU}(N)_{k} \leftrightarrow \mathrm{U}(k)_{-N,-N},
$$

and another useful level-rank duality relates

$$
\mathrm{U}(N)_{k, k+N} \leftrightarrow \mathrm{U}(k)_{-N,-k-N} .
$$

In particular, the $\mathrm{SU}(N)$ theory maps to the simplest $\mathrm{U}(k)$ theory, that has equal levels for its two factors.

Using (2.1) (and the fact that there is no shift of $\mathrm{U}(1)$ levels), it is easy to express the same dualities in the dimensional regularization conventions. To avoid confusion we use hatted indices for these conventions, and then we have

$$
\mathrm{SU}(\hat{N})_{\hat{k}} \leftrightarrow \mathrm{U}(\hat{k}-\hat{N})_{-\hat{k},-\hat{N}}
$$

and

$$
\mathrm{U}(\hat{N})_{\hat{k}, \hat{k}} \leftrightarrow \mathrm{U}(\hat{k}-\hat{N})_{-\hat{k},-\hat{k}}
$$

In this convention the second level-rank duality relates the simplest $\mathrm{U}(\hat{N})$ theories.

Consider now the duality of [1-3] relating $\mathrm{U}(N)$ Chern-Simons theories coupled to $N_{\mathrm{f}}$ fermions in the fundamental representation, to Chern-Simons theories coupled to $N_{\mathrm{f}}$ scalars in the fundamental representation (the duality also involves a Legendre transform with respect to the singlet scalar operator in one of the theories, but this is not important for any of the issues we discuss in this note). In the large $N$ limit, the 't Hooft coupling in the dimensional regularization procedure is defined by $\lambda \equiv \hat{N} / \hat{k}$, and in the Yang-Mills regularization by $\lambda \equiv N /(k+N \operatorname{sign}(k))$. In both cases $-1<\lambda<1$, and in the large $N$ limit it is effectively a continuous variable. Various quantities were computed exactly as a function of this 't Hooft coupling in the large $N$ limit of these non-supersymmetric field theories [3, 10, 20, 23, 25-37], and were all found to be consistent with the duality.

In [3] it was argued that the precise form of this duality for finite $N$ could be determined by deforming the fermionic theory by a fermion mass such that it flows at low energies to a pure CS theory (depending on the sign of the mass), and mapping that deformation to the scalar side where one obtains a different pure CS theory (that theory again depends on the sign of the mass, because for one sign there is a scalar condensate partly breaking the gauge group). The two low-energy CS theories must then be related by level-rank duality. The arguments of [3] were not careful about the precise form of the level-rank duality, and about whether we have an $\mathrm{SU}(N)$ or $\mathrm{U}(N)$ gauge group, and they found that (in the Yang-Mills regularization) the duality should relate the theory of $N_{\mathrm{f}}$ fundamental

\footnotetext{
${ }^{1}$ Here and in some places below we assume without loss of generality that $k>0$. The results for $k<0$ follow by a parity transformation.
} 
scalars coupled to $\mathrm{U}(N)_{k}$, to a theory of $N_{\mathrm{f}}$ fundamental fermions coupled to $\mathrm{U}(k)_{N_{\mathrm{f}} / 2-N}$. Using the precise form of the level-rank dualities above, we see that there are actually three possible dualities that are consistent with level-rank duality:

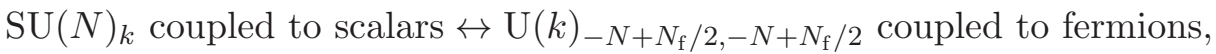

$$
\begin{aligned}
& \mathrm{U}(N)_{k, k} \text { coupled to scalars } \leftrightarrow \mathrm{SU}(k)_{-N+N_{\mathrm{f}} / 2} \text { coupled to fermions, } \\
& \mathrm{U}(N)_{k, k+N} \text { coupled to scalars } \leftrightarrow \mathrm{U}(k)_{-N+N_{\mathrm{f}} / 2,-N-k+N_{\mathrm{f}} / 2} \text { coupled to fermions. }
\end{aligned}
$$

The evidence so far for the validity of these dualities is identical, since it is just at leading order in the large $N$ limit, where the differences between them are negligible. We conjecture that they are all correct. The main difference between the different theories is in the baryonic and monopole operators, and we will discuss these operators and their consistency with the duality in the next sections.

It was argued in [28] that when the $\mathrm{U}(N)$ Chern-Simons-matter theories are compactified on a circle, the logarithms of the eigenvalues of their holonomies are quantized to be an integer multiple of $2 \pi / \hat{k}$. In the $\mathrm{SU}(N)$ theory the eigenvalues sum up to zero (up to an overall shift by a multiple of $2 \pi$ ), and the quantization applies to the difference between any pair of eigenvalues. This quantization has important effects for the thermodynamical behavior of these theories, which was analyzed in the large $N$ limit in [28, 29] (following [1]).

Note that the $\mathrm{SU}(N)$ theory has a $\mathrm{U}(1)$ baryon-number symmetry, carried by the fields in the fundamental representation. The $\mathrm{U}(N)$ theories also have a $\mathrm{U}(1)$ global symmetry, which is a combination of the baryon-number symmetry and the "topological" symmetry generated by the current $J^{\mu}=\epsilon^{\mu \nu \rho} \operatorname{tr}\left(F_{\nu \rho}\right)$. One combination of these two currents is gauged, and any other combination is a global symmetry, that maps under the duality to the corresponding global symmetry on the other side. The precise combination that is gauged depends on the Chern-Simons level of the U(1) gauge field; it is independent of the $\mathrm{SU}(N)$ Chern-Simons level.

Let us end this section by clarifying the relation of these dualities to dualities between $\mathcal{N}=2$ supersymmetric CS-matter theories [11-14]. For these theories the Yang-Mills regularization, which preserves supersymmetry, is usually used. However, the supersymmetric pure Yang-Mills-Chern-Simons theory contains two extra massive gluinos in addition to the pure CS theory. Integrating these out gives a shift between the level of the $\mathcal{N}=2$ supersymmetric $\mathrm{SU}(N)$ CS theory and the non-supersymmetric one, of the form

$$
k^{\mathcal{N}=2}=k^{\mathcal{N}=0}+N \operatorname{sign}\left(k^{\mathcal{N}=0}\right) .
$$

This is precisely of the same form as (2.1), though their origin is completely different (a loop involving gluons in one case, a loop with two gluinos in the other). Thus, the levelrank dualities for pure $\mathcal{N}=2$ CS theories take the same form as (2.4) and (2.5) above. This is consistent with the form of the known supersymmetric dualities between ChernSimons-matter theories with chiral multiplets in the fundamental and/or anti-fundamental representation [11-14]; in fact taking $N_{\mathrm{f}} \rightarrow 0$ in the equation for the mapping between the two sides of these dualities gives precisely the mappings (2.4) and (2.5). When we flow from $\mathcal{N}=2$ supersymmetric Yang-Mills-Chern-Simons-matter dualities to non-supersymmetric 
CS-matter dualities, as described in $[10,20]$, both for the $\mathrm{SU}(N) \leftrightarrow \mathrm{U}(\tilde{N})$ dualities and for the $\mathrm{U}(N) \leftrightarrow \mathrm{U}(\tilde{N})$ dualities, we recover (2.6)-(2.8), taking into account the shift in the Chern-Simons level arising from integrating out massive fermions. Note that apriori $\mathcal{N}=2$ Chern-Simons-matter theories exist for any value of $k^{\mathcal{N}=2}$, but (2.9) suggests that if we try to flow from there to $\mathcal{N}=2$ supersymmetric pure Chern-Simons theories by giving masses to all the flavors, we will only obtain theories with $\left|k^{\mathcal{N}=2}\right|>N$ (in other cases this flow leads to supersymmetry breaking). This is consistent with all cases we are familiar with.

\section{Baryons and monopoles in Chern-Simons-matter theories}

In the large $N$ limit, the finite-dimension operators in all the theories discussed in the previous section are the same. They are just the bi-fundamental operators, schematically $\Phi^{\dagger} D D \cdots D \Phi$ or $\bar{\psi} D D \cdots D \psi$ (these include currents of all spins, that are conserved in the large $N$ limit for any value of $\lambda$ ), and their "multi-trace" products. However, operators whose dimensions scale with $N$ are significantly different in the different theories discussed above, and we review their properties in this section.

Let us begin with the $\mathrm{SU}(N)$ theories, and for simplicity let us take a single matter field in the fundamental representation, $N_{\mathrm{f}}=1$ (the generalization to arbitrary values of $N_{\mathrm{f}}$ is straightforward; for $N_{\mathrm{f}}=1$ the CS level in CS-fermion theories must be a half-integer). In such theories we can form baryon operators by taking a product of $N$ fields in the fundamental representation and anti-symmetrizing their color indices. We normalize the $\mathrm{U}(1)$ global symmetry such that the baryon operator carries charge 1 .

If we have a fermion in the fundamental representation, we can just take a product of its $N$ color components and anti-symmetrize them, and this gives the lowest-dimension operator carrying baryon-number charge. This operator has a classical dimension $\Delta=N$; of course this dimension can obtain quantum corrections. Note that the fermions have two different spin states, and each fermion can be independently chosen to be in each of these states, so we actually have $2^{N}$ baryon operators of this type, of various spins ranging up to $N / 2$.

For scalars in the fundamental representation we cannot do this since the $N$ bosonic operators that we multiply must all be different in order for the baryon operator to be non-zero. So to form baryon operators we need to take some derivative operator acting on each color component of the scalar, with a different derivative operator for each component. Given that the scalar obeys (in the free theory) $\partial^{2} \phi=0$, the number of different operators with $j$ derivatives acting on the scalar is $2 j+1$. Choosing the minimal number of derivatives that are all different, we find that the minimal dimension baryonic operator that we can form from scalars has a dimension that scales as $\Delta \simeq \frac{2}{3} N^{3 / 2}$ for large $N$ [38]. Again there are many operators of this type, of different spins, depending on precisely how we contract the different derivatives.

In $\mathrm{U}(N)$ theories baryons are not gauge-invariant, but a similar role is played by monopole operators. These operators are characterized by a gauge flux around them, which can be chosen to be in the Cartan subalgebra of the gauge group. When the gauge group is $\mathrm{U}(N)$ (rather than $\mathrm{SU}(N) \times \mathrm{U}(1)$ ), the monopole operators are characterized by $N$ integers 
$q_{i}$ (up to permutations in the Weyl group), which are the magnetic fluxes in $\mathrm{U}(1)^{N} \subset$ $\mathrm{U}(N)$ (these are known as the GNO charges). We normalize the global "topological" $\mathrm{U}(1)$ charge so that this monopole operator carries a global charge $\sum_{i=1}^{N} q_{i}$. Note that monopole operators with different magnetic fluxes that carry the same global charge can mix with each other in the quantum theory.

In a Yang-Mills theory these monopoles are gauge-invariant operators, but in a (YangMills-)Chern-Simons theory the Chern-Simons coupling gives the monopole operators an electric charge. The simplest possibility to analyze is the $\mathrm{U}(N)_{k, k}$ theory. In this theory a generic monopole operator, with $n$ different non-zero charges $q_{i}$, breaks the gauge group to $\mathrm{U}(1)^{n} \times \mathrm{U}(N-n)$, and carries a charge $q_{i} k$ under the $\mathrm{U}(1)_{i}$ unbroken gauge group. This charge must be balanced by an appropriate number of fields in the (anti-)fundamental representation of $\mathrm{U}(N)$ in order to obtain a gauge-invariant operator. Note that $k$ here is defined in the Yang-Mills regularization, since the monopoles are naturally defined in the high-energy Yang-Mills-Chern-Simons theory.

Let us consider for simplicity the monopole operators with charges $\left\{q_{i}\right\}=(1,0, \cdots, 0)$. These are expected to be the lowest-dimension operators carrying the global U(1) charge. In this case we need to multiply the monopole by $|k|$ fields in the fundamental or antifundamental representation, which all carry the same gauge group index. In the scalar theory we can just put in $|k|$ scalars, so the dimension of the corresponding operator is classically $\Delta=|k| \Delta_{\text {scalar }}+\Delta_{\text {monopole }}$, where $\Delta_{\text {monopole }}$ is the dimension of the monopole operator itself (this can be thought of as the difference in energy between the lowest state with flux $\left\{q_{i}\right\}=(1,0, \cdots, 0)$ on $S^{2}$, before adding the necessary fields to make it gauge-invariant, and the lowest zero-flux state). This difference vanishes in the non-supersymmetric CSmatter theory with no fluxes for background fields; in theories with background fluxes (which is usually the case in supersymmetric theories) it can be non-zero. $\Delta_{\text {scalar }}$ here is the dimension of the scalar in a monopole background, which is the same as the energy of the lightest scalar state on $S^{2}$ in the flux background. ${ }^{2}$ For unit flux it is $\Delta_{\text {scalar }}=1$; note that this is shifted from the lowest energy without the flux, that gives $\Delta_{\text {scalar }}=\frac{1}{2}$. More over, this scalar state carries spin one half [39], such that we have $2^{|k|}$ states of this type, with $\Delta=|k|$ (classically), carrying different spins up to $|k| / 2$.

In the fermionic theory, as in our discussion of the baryons above, we need to choose $|k|$ different fermionic operators multiplying the monopole in order for the resulting gaugeinvariant operator to be non-zero. Thus, as in our analysis above, the classical dimension of the monopole operator in the large $N$ limit is $\Delta \simeq \frac{2}{3}|k|^{3 / 2}$. Note that there is also a contribution here from the fermion operators (involving the dimension of the fermion operators in the monopole background), but this is negligible compared to the contribution from the derivatives. The lowest fermion state in the monopole background carries spin zero [39], but the gauge-invariant monopole operators we obtain can have many different spins, depending on precisely how we contract the indices on the derivatives.

If we have different levels for the $\mathrm{SU}(N)$ and the $\mathrm{U}(1)$ the analysis changes. Let us first discuss the "bare" monopole operators for $\mathrm{U}(N)_{k, k^{\prime}}$ theories, assuming $k, k^{\prime}>0$. It

\footnotetext{
${ }^{2}$ The discussion here is not completely rigorous, since the monopole separately and the scalar separately are not gauge-invariant. However, it is valid at least at weak coupling (and in supersymmetric theories for chiral operators), which is enough for our purposes here.
} 
is convenient to decompose the charge of the lowest monopole into its $\mathrm{U}(1)$ and $\mathrm{SU}(N)$ components:

$$
\left\{q_{i}\right\}=(1,0, \cdots, 0)=\left(\frac{1}{N}, \frac{1}{N}, \cdots, \frac{1}{N}\right)+\left(\frac{N-1}{N},-\frac{1}{N}, \cdots,-\frac{1}{N}\right) .
$$

When the $\mathrm{U}(1)$ has CS level $k^{\prime}$, the $\mathrm{U}(1)$ electric charge of this monopole is equal to $k^{\prime}$ times that of the fundamental representation. When the $\mathrm{SU}(N)$ has $\mathrm{CS}$ level $k$, the $\mathrm{SU}(N)$ electric charge is in the symmetric product of $k$ fundamental representations of $\mathrm{SU}(N)$. Thus, when the levels are equal, this charge is the same as that of $k$ fields in the fundamental representation of $\mathrm{U}(N)$, and the monopole can be made neutral by multiplying it by $k$ fields in the anti-fundamental representation, as above.

When $k^{\prime}$ is not equal to $k$, the electric charge we obtain is only a consistent charge in $\mathrm{U}(N)$ when $k-k^{\prime}=0(\bmod N)$, a constraint that is presumably required for gaugeinvariance of the $\mathrm{U}(N)$ Chern-Simons term. ${ }^{3}$ If (say) $k^{\prime}=k+N$ (the case that appears in (2.8)) then the monopole carries $N$ extra units of $\mathrm{U}(1)$ charge in the fundamental representation compared to the product of $k$ fields in the fundamental representation of $\mathrm{U}(N)$. Thus its charges are the same as an $\mathrm{SU}(N)$ baryon added to that product (and it can be made neutral by multiplying it by an anti-baryon and $k$ fields in the anti-fundamental representation). The fundamental fields that we need to add to make the monopole gaugeinvariant obey similar symmetry constraints to the ones above; similar to monopoles for the fields with color index one, and similar to baryons for the fields with other color indices. Thus, in the scalar theory we obtain by the same analysis as above a classical dimension of order $\frac{2}{3} N^{3 / 2}$ at large $N$, and in the fermionic theory we obtain a classical dimension of order $\frac{2}{3}|k|^{3 / 2}$ for this minimal globally-charged operator in the $\mathrm{U}(N)_{k, k+N}$ theory. The generalization to other values of $\left(k^{\prime}-k\right)$ is straightforward; if $k^{\prime}=k+n N$ for some integer $n$, the lowest-dimension operator carrying the minimal global $\mathrm{U}(1)$ charge would look like a bound state of a monopole and $n$ baryons.

\section{Mapping of baryons and monopoles under the duality}

Dualities between different Chern-Simons-matter theories should map the lowest-dimension operator with some spin and global charge to the lowest-dimension operator with the same spin and global charge in the dual theory. For dualities between $\mathrm{SU}(N)$ and $\mathrm{U}(\tilde{N})$ theories, this means that baryons should be mapped to monopoles, and this is indeed what happens for chiral monopole and baryon operators in supersymmetric dualities of this type [13, 14].

In the non-supersymmetric case, let us start from the duality (2.7). In the fermionic $\mathrm{SU}(k)_{-N+1 / 2}$ theory the globally charged operators are baryons, with a lowest dimension $|k|$ at weak coupling. In the bosonic $\mathrm{U}(N)_{k, k}$ theory, the globally charged operators are monopoles, and their lowest classical dimension is $|k|$. Thus, there is an exact match between the classical dimensions on both sides (even at finite $N$ and $k$ ). This suggests that these dimensions do not acquire quantum corrections (at large $N$, and maybe even at

\footnotetext{
${ }^{3}$ Here it is important that the gauge group is precisely $\mathrm{U}(N)=(\mathrm{SU}(N) \times \mathrm{U}(1)) / Z_{N}$; we will not discuss other quantizations of the $\mathrm{U}(1)$ charge here.
} 
finite $N$ ), even though we do not know any reason for this. Note that on both sides we have $2^{|k|}$ operators of different spins, in agreement with the duality. For $N_{\mathrm{f}}>1$ one can check that the global $\mathrm{SU}\left(N_{\mathrm{f}}\right)$ quantum numbers of the baryons and monopoles also match between the two sides.

In the duality (2.6), the lightest globally-charged operators in the bosonic $\mathrm{SU}(N)_{k}$ theory are baryons, whose dimension at weak coupling scales as $\Delta \simeq \frac{2}{3} N^{3 / 2}$. In the fermionic $\mathrm{U}(k)_{-N+1 / 2,-N+1 / 2}$, the lowest-dimension charged operators are monopoles, whose dimension in the large $N$ limit scales as $\Delta \simeq \frac{2}{3} N^{3 / 2}$. So again we find a precise match of the classical dimensions in the large $N$ limit. In both cases the degeneracy and spins arise from the different ways of contracting derivatives acting on spin zero operators, so these also match between the two sides, as do the $\mathrm{SU}\left(N_{\mathrm{f}}\right)$ representations when $N_{\mathrm{f}}>1$.

It was argued in [23] that the monopole dimension in the fermionic theory at large $N$ is independent of the 't Hooft coupling $\lambda$, and thus that the result above $\Delta \simeq \frac{2}{3} N^{3 / 2}$ is actually valid for any value of $\lambda$. The reasons for this absence of quantum corrections are not completely clear (it was argued in [40] that general diagrams would contribute corrections to $\Delta$ that could even change its large $N$ scaling), but it follows from the thermal partition function computation of [23]. The duality then suggests that also the dimensions of the baryons in CS-scalar theories do not receive quantum corrections in the large $N$ limit; again there is no obvious reason for this to be true.

Note that the analysis of [23] was naively done using the dimensional regularization convention, with the eigenvalues of the holonomies of the $\mathrm{U}(N)_{k, k}$ theory on a circle separated by distances $2 \pi /|\hat{k}|$. Here the relevant level is the $\mathrm{SU}(N)$ level, so the computation in [23] was done for an $\mathrm{SU}(N)$ level $|k|=|\hat{k}|-N$. Dimensional regularization was indeed used in [23] (following [28, 29]) to define the level in the computation of the eigenvalue distribution (which here involves the $(N-1)$ eigenvalues that do not have any background monopole flux). However, the contribution to the dimension of the monopole operator in [23] actually comes from the integration over the single eigenvalue corresponding to the $\mathrm{U}(1)$ subgroup in which the monopole flux was turned on. This integration was performed using the fact that the monopole background with charges $\left\{q_{i}\right\}$ induces $k q_{i}$ units of background charge for the $i$ 'th $\mathrm{U}(1)$ gauge group. As we discussed above, this is true for the $\mathrm{U}(N)_{k, k}$ theory where $k$ is defined using the Yang-Mills regularization. In the analysis of [23] the result only depends on $k$, going as (for the simplest monopole) $\Delta \simeq \frac{2}{3}|k|^{3 / 2}$ (independently of $N$ ). Thus, this analysis should be interpreted as being done in the $\mathrm{U}(N)_{k, k}$ theory (whose dimensional regularization $\mathrm{SU}(N)$ CS level is $\hat{k}=k+N \operatorname{sign}(k)$ ). Therefore, our statement in the previous paragraph about the implications of the analysis of [23] is indeed correct in the fermionic $\mathrm{U}(k)_{-N+1 / 2,-N+1 / 2}$ theory that we mentioned there.

In the duality (2.8), we saw in the previous section that the classical dimension of the lightest globally-charged state is the sum of the dimensions for baryons and monopoles. So by joining together our analysis of the two previous cases, these lightest states map to each other also under this duality (2.8), again assuming that the dimensions of the corresponding operators have no corrections in the large $N$ limit.

In the supersymmetric Chern-Simons-matter dualities, we can explicitly map the chiral baryon or monopole operators, since we can read off their dimensions from the supersym- 
metric index [41-43]. This mapping was not yet performed for $\mathrm{SU}(N)$ dualities, but it was performed for some dualities between $\mathrm{U}(N)$ CS-matter theories in [40]. Obviously, since the partition functions are the same on both sides, the monopole operators were also found to match. However, surprisingly, it was found that the dimensions of the lowest chiral monopole operators are often much larger than the dimensions computed above (the relevant dimensions are the ones for theories with scalar fields, since such fields are present in the supersymmetric theories), and can even scale differently with $N$. This implies that in these theories the dimensions of the monopole operators that classically have the lowest dimensions are usually not protected. The analysis above suggests that the anomalous dimensions of at least some of these operators are negligible, at least in the large $N$ limit, but it is not known how to test this directly.

\section{$5 \quad$ Mapping to high-spin gravities}

The $\mathrm{U}(N)_{k}$ Chern-Simons-matter theories discussed above are conjectured [1-3, 9] (following the $k=0$ case discussed in $[4,5])$ to correspond to Vasiliev's high-spin gravity theories on $A d S_{4}$. For $N_{\mathrm{f}}=1$ they are mapped to the original Vasiliev theory, while for higher $N_{\mathrm{f}}$ they map to a version of this theory where all the fields transform in the adjoint representation of $\mathrm{U}\left(N_{\mathrm{f}}\right)$. The coupling constant of the Vasiliev theories in this mapping goes as $1 / N$ (see a more detailed discussion below), and since these theories are only understood classically, this duality is well-defined only in the large $N$ limit; the Chern-Simons-matter theories can be viewed as giving a non-perturbative completion of the Vasiliev theories. In a special supersymmetric case the string theory completion of the Vasiliev theory is also known (at least formally) [9], so this string theory can also be viewed as a UV completion (identical to the one provided by the CS-matter theory).

Since the duality is only understood at large $N$, it is not clear which, if any, of the CS-matter theories discussed in the previous sections corresponds to the Vasiliev theory. In fact, they all do. This is because we can move between the different CS-matter theories by shifting the U(1) level and gauging or "ungauging" the U(1), and in the context of the Vasiliev theory, as discussed extensively in [9] (following [44]), these transformations just involve a modification of the boundary conditions of the $4 d \mathrm{U}(1)$ gauge field of the Vasiliev theory. Note that this is consistent with the fact that the operators in these theories whose dimension does not scale with $N$ are identical.

In particular, let us begin with the Vasiliev theory corresponding to the $\mathrm{SU}(N)_{k}$ theory coupled to scalars (appearing in (2.6)). This theory (up to the $\mathrm{U}(1)$ issues) is believed to be dual to the Vasiliev theory with a theta angle related to the $\mathrm{SU}(N)$ 't Hooft coupling by $\theta=\frac{\pi}{2} \lambda[3,9,45]$. This Vasiliev theory includes a $4 d \mathrm{U}(1)$ gauge field, which is related in the usual way to the global baryon number symmetry in the $3 d \mathrm{SU}(N)$ CS-matter theory; thus the charged states in the bulk should include the baryons of this theory. As discussed above, the same theory should have a description as a $\mathrm{U}(k)_{-N,-N}$ theory coupled to fermions, and in that description the same charged states are monopoles of the CS-matter theory.

We can take this theory and modify the boundary conditions on the $4 d \mathrm{U}(1)$ gauge field, in a way that corresponds to gauging the $\mathrm{U}(1)$ global symmetry of the $\mathrm{SU}(N)$ CS-matter 
theory. This involves making the boundary value of the $4 d \mathrm{U}(1)$ gauge field dynamical, instead of the subleading term in the expansion of this gauge field near the boundary [44]; one should also be careful about the charge-quantization conditions to ensure that the resulting theory has gauge group $\mathrm{U}(N)$ rather than $\mathrm{SU}(N) \times \mathrm{U}(1)$. We can then add for this $3 d$ gauged $\mathrm{U}(1)$ a CS term of level $k^{\prime}$ (which has no relation to $k$ ). For specific choices of $k^{\prime}$ this gives the theories appearing in (2.7) and (2.8) above, and our discussion above implies that the interpretation of this procedure on the fermionic side is as changing the theory on the right-hand side of (2.6) to that on the right-hand side of (2.7) or (2.8). Note that if we start from a $\mathrm{U}(N)$ gauge theory and gauge its global "topological" $\mathrm{U}(1)$ symmetry, we generally get a $\mathrm{U}(N) \times \mathrm{U}(1)$ theory, with a mixed CS coupling between the $\mathrm{U}(1) \subset \mathrm{U}(N)$ and the new $\mathrm{U}(1)$. However, in some cases this turns out to be equivalent to an $\mathrm{SU}(N)$ theory (see, for instance, the discussion in [13]; this is called "ungauging"), and presumably this also happens here in the fermionic theory when we go from (2.6) or (2.8) to $(2.7)$.

As discussed in [44], the operation of gauging a $3 d \mathrm{U}(1)$ global symmetry maps by the AdS/CFT correspondence to an electric-magnetic duality on the $4 d$ bulk gauge field, and the operation of shifting the Chern-Simons level of this $3 d$ gauged $\mathrm{U}(1)$ is the same as shifting the bulk theta angle (multiplying $F \wedge F)^{4}$ by a multiple of $2 \pi$ (this has no effect on the bulk physics, but does affect the physics in the presence of the boundary). So all the operations described in the previous paragraph can be interpreted as $\operatorname{SL}(2, Z)$ transformations on the bulk $\mathrm{U}(1)$ gauge theory. In particular, if in the $\mathrm{SU}(N)$ picture the allowed charges of states in the bulk were electric charges of the bulk U(1) (with the total $4 d$ magnetic charge vanishing because of the boundary conditions), after these operations the only allowed charges are dyonic (with the ratio of the electric and magnetic charge depending on $k^{\prime}$ ).

As we saw in the previous section, this modification of the allowed charges is mirrored in the gauge theory by the fact that instead of having baryon operators we now have monopole operators (that also carry some baryon number charge). The monopole charge of the CS-matter theory should thus be viewed as the electric-magnetic dual in the bulk of the baryon number charge.

At first sight we have a contradiction, since on one hand the only difference between the theories (2.6), (2.7) and (2.8) on the gravity side is in the boundary conditions, but on the other hand their spectrum of operators is completely different. However, the bulk theory in all these cases is actually exactly the same, and it should thus contain excitations corresponding to all the baryon and monopole operators that we discussed above. From the bulk point of view, the reason for the different spectrum of these theories is that the different boundary conditions result in different Gauss' law constraints on the total charge. The constraint for the $\mathrm{SU}(N)$ theory allows only non-zero total electric (= baryon number) charge, while the one of $\mathrm{U}(N)$ theories allows only a specific dyonic charge (which we called monopole number). So, while there are bulk excitations corresponding to both baryons

\footnotetext{
${ }^{4}$ Here we refer to the theta angle of the $\mathrm{U}(1)$ gauge theory on $A d S_{4}$. This should not be confused with the theta angle mentioned elsewhere in this section, that appears in the equations of motion of the Vasiliev theory.
} 
and monopoles, only a subset of the multi-baryon and multi-monopole states, which obeys Gauss' law, gives consistent states in the full theory. The allowed charge in all cases maps to the global U(1) charge of the $3 d$ Chern-Simons-matter theory.

If we interpret the Vasiliev theory from the point of view of the scalar theory, then the baryons have a dimension going as $N^{3 / 2}$, while the monopoles have a dimension scaling as $|k|$; from the fermionic point of view the same numbers are $|k|^{3 / 2}$ and $N$.

The action for the Vasiliev theory is not yet known, but only its equations of motion, which determine the action up to an overall scaling. This scaling may be determined, for instance, from the two-point function of the energy-momentum tensor in the CS-matter theory, which should be related to the Einstein term in the bulk action (it is not affected by the theta parameter). This two-point function is proportional in the scalar interpretation to $N \frac{\sin (\pi \lambda)}{\pi \lambda}$ [3], which for any $-1<\lambda<1$ in the 't Hooft limit scales as $N$.

Thus, from the point of view of the scalars, the dimension of the monopole operators $\Delta \simeq|k|$ is consistent with the monopoles being classical solutions of the Vasiliev theory in the bulk or of its UV completion, which carry a magnetic U(1) charge in our conventions above (though it does not prove that such classical solutions exist). These solutions can be thought of as charged black holes or charged solitons in the Vasiliev theory. The discussion above implies that the energy of these classical solutions, in units set by the Vasilliev gravitational coupling, should depend on the theta angle appearing in the Vasiliev equations of motion. In particular it diverges (in these units) in the $\theta \rightarrow 0$ limit. It would be very interesting to find such classical solutions, if they exist, in order to confirm this picture.

On the other hand, the baryons (from the scalar point of view) with $\Delta \simeq N^{3 / 2}$ should be much heavier than this scale, and cannot be viewed as finite excitations on top of the classical Vasiliev theory. So there should not be any classical electrically-charged solutions.

The generalization of all our discussions to the supersymmetric Chern-Simons-matter theories, whose gravitational duals were discussed in [9], seems to be straightforward, and we will not perform it here. The main difference is that in these theories, since they include both scalars and fermions in the fundamental representation, the dimensions of the lowestdimension baryons and monopoles scale as $N$ and $|k|$, respectively, so both of these may correspond to classical solutions. For theories with $\mathcal{N} \geq 2$ supersymmetry, the precise dimensions of the lowest-dimensional chiral baryon or monopole operators may be found along the lines of [40]. These could correspond to classical solutions of the bulk theory that preserve some of the supersymmetry.

\section{Acknowledgments}

I would like to thank Guy Gur-Ari, Zohar Komargodski and Tarun Sharma for useful discussions, and especially Djordje Radičević for useful discussions and comments on a draft of this manuscript. This work was supported in part by an Israel Science Foundation center for excellence grant (grant no. 1989/14), by the Minerva foundation with funding from the Federal German Ministry for Education and Research, by the I-CORE program of the Planning and Budgeting Committee and the Israel Science Foundation (grant number 1937/12), by the Henry Gutwirth Fund for Research, and by the ISF within the ISF-UGC 
joint research program framework (grant no. 1200/14). OA is the incumbent of the Samuel Sebba Professorial Chair of Pure and Applied Physics.

Open Access. This article is distributed under the terms of the Creative Commons Attribution License (CC-BY 4.0), which permits any use, distribution and reproduction in any medium, provided the original author(s) and source are credited.

\section{References}

[1] S. Giombi, S. Minwalla, S. Prakash, S.P. Trivedi, S.R. Wadia and X. Yin, Chern-Simons Theory with Vector Fermion Matter, Eur. Phys. J. C 72 (2012) 2112 [arXiv:1110.4386] [INSPIRE].

[2] O. Aharony, G. Gur-Ari and R. Yacoby, $D=3$ Bosonic Vector Models Coupled to Chern-Simons Gauge Theories, JHEP 03 (2012) 037 [arXiv:1110.4382] [INSPIRE].

[3] O. Aharony, G. Gur-Ari and R. Yacoby, Correlation Functions of Large- $N$ Chern-Simons-Matter Theories and Bosonization in Three Dimensions, JHEP 12 (2012) 028 [arXiv: 1207.4593] [INSPIRE].

[4] I.R. Klebanov and A.M. Polyakov, AdS dual of the critical $O(N)$ vector model, Phys. Lett. B 550 (2002) 213 [hep-th/0210114] [INSPIRE].

[5] E. Sezgin and P. Sundell, Holography in 4D (super) higher spin theories and a test via cubic scalar couplings, JHEP 07 (2005) 044 [hep-th/0305040] [INSPIRE].

[6] M.A. Vasiliev, More on equations of motion for interacting massless fields of all spins in (3+1)-dimensions, Phys. Lett. B 285 (1992) 225 [INSPIRE].

[7] J.M. Maldacena and A. Zhiboedov, Constraining Conformal Field Theories with A Higher Spin Symmetry, J. Phys. A 46 (2013) 214011 [arXiv:1112.1016] [inSPIRE].

[8] J.M. Maldacena and A. Zhiboedov, Constraining conformal field theories with a slightly broken higher spin symmetry, Class. Quant. Grav. 30 (2013) 104003 [arXiv: 1204.3882] [INSPIRE].

[9] C.-M. Chang, S. Minwalla, T. Sharma and X. Yin, ABJ Triality: from Higher Spin Fields to Strings, J. Phys. A 46 (2013) 214009 [arXiv:1207.4485] [InSPIRE].

[10] S. Jain, S. Minwalla and S. Yokoyama, Chern Simons duality with a fundamental boson and fermion, JHEP 11 (2013) 037 [arXiv:1305.7235] [INSPIRE].

[11] A. Giveon and D. Kutasov, Seiberg Duality in Chern-Simons Theory, Nucl. Phys. B 812 (2009) 1 [arXiv:0808.0360] [INSPIRE].

[12] F. Benini, C. Closset and S. Cremonesi, Comments on 3d Seiberg-like dualities, JHEP 10 (2011) 075 [arXiv:1108.5373] [INSPIRE].

[13] O. Aharony, S.S. Razamat, N. Seiberg and B. Willett, $3 d$ dualities from $4 d$ dualities, JHEP 07 (2013) 149 [arXiv: 1305.3924] [INSPIRE].

[14] O. Aharony and D. Fleischer, IR Dualities in General 3d Supersymmetric $\mathrm{SU}(N) Q C D$ Theories, JHEP 02 (2015) 162 [arXiv:1411.5475] [INSPIRE].

[15] S.G. Naculich, H.A. Riggs and H.J. Schnitzer, Group Level Duality in WZW Models and Chern-Simons Theory, Phys. Lett. B 246 (1990) 417 [InSPIRE]. 
[16] M. Camperi, F. Levstein and G. Zemba, The Large-N Limit of Chern-Simons Gauge Theory, Phys. Lett. B 247 (1990) 549 [INSPIRE].

[17] E.J. Mlawer, S.G. Naculich, H.A. Riggs and H.J. Schnitzer, Group level duality of WZW fusion coefficients and Chern-Simons link observables, Nucl. Phys. B 352 (1991) 863 [INSPIRE].

[18] T. Nakanishi and A. Tsuchiya, Level rank duality of WZW models in conformal field theory, Commun. Math. Phys. 144 (1992) 351 [InSPIRE].

[19] S.G. Naculich and H.J. Schnitzer, Level-rank duality of the $\mathrm{U}(N)$ WZW model, Chern-Simons theory and 2d qYM theory, JHEP 06 (2007) 023 [hep-th/0703089] [INSPIRE].

[20] G. Gur-Ari and R. Yacoby, Three Dimensional Bosonization From Supersymmetry, JHEP 11 (2015) 013 [arXiv:1507.04378] [INSPIRE].

[21] S. Pufu, private communication.

[22] S. Pufu, presentation at http://online.kitp.ucsb.edu/online/qft-c14/pufu/, (2014).

[23] Đ. Radičević, Disorder Operators in Chern-Simons-Fermion Theories, arXiv:1511.01902 [INSPIRE].

[24] W. Chen, G.W. Semenoff and Y.-S. Wu, Two loop analysis of nonAbelian Chern-Simons theory, Phys. Rev. D 46 (1992) 5521 [hep-th/9209005] [INSPIRE].

[25] S. Banerjee, S. Hellerman, J. Maltz and S.H. Shenker, Light States in Chern-Simons Theory Coupled to Fundamental Matter, JHEP 03 (2013) 097 [arXiv:1207.4195] [INSPIRE].

[26] G. Gur-Ari and R. Yacoby, Correlators of Large-N Fermionic Chern-Simons Vector Models, JHEP 02 (2013) 150 [arXiv:1211.1866] [INSPIRE].

[27] Đ. Radičević, Singlet Vector Models on Lens Spaces, JHEP 03 (2014) 048 [arXiv: 1210.0255] [INSPIRE].

[28] O. Aharony, S. Giombi, G. Gur-Ari, J.M. Maldacena and R. Yacoby, The Thermal Free Energy in Large-N Chern-Simons-Matter Theories, JHEP 03 (2013) 121 [arXiv:1211.4843] [INSPIRE].

[29] S. Jain, S. Minwalla, T. Sharma, T. Takimi, S.R. Wadia and S. Yokoyama, Phases of large- $N$ vector Chern-Simons theories on $S^{2} \times S^{1}$, JHEP 09 (2013) 009 [arXiv:1301.6169] [INSPIRE].

[30] T. Takimi, Duality and higher temperature phases of large- $N$ Chern-Simons matter theories on $S^{2} \times S^{1}$, JHEP 07 (2013) 177 [arXiv: 1304.3725] [INSPIRE].

[31] Y. Frishman and J. Sonnenschein, Breaking conformal invariance - Large- $N$ Chern-Simons theory coupled to massive fundamental fermions, JHEP 12 (2013) 091 [arXiv:1306.6465] [INSPIRE].

[32] W.A. Bardeen and M. Moshe, Spontaneous breaking of scale invariance in a $D=3 \mathrm{U}(N)$ model with Chern-Simons gauge fields, JHEP 06 (2014) 113 [arXiv:1402.4196] [INSPIRE].

[33] S. Jain, M. Mandlik, S. Minwalla, T. Takimi, S.R. Wadia and S. Yokoyama, Unitarity, Crossing Symmetry and Duality of the S-matrix in large- $N$ Chern-Simons theories with fundamental matter, JHEP 04 (2015) 129 [arXiv:1404.6373] [INSPIRE].

[34] Y. Frishman and J. Sonnenschein, Large- $N$ Chern-Simons with massive fundamental fermions - A model with no bound states, JHEP 12 (2014) 165 [arXiv:1409.6083] [INSPIRE]. 
[35] M. Moshe and J. Zinn-Justin, 3D Field Theories with Chern-Simons Term for Large- $N$ in the Weyl Gauge, JHEP 01 (2015) 054 [arXiv: 1410.0558] [INSPIRE].

[36] A. Bedhotiya and S. Prakash, A test of bosonization at the level of four-point functions in Chern-Simons vector models, JHEP 12 (2015) 032 [arXiv: 1506.05412] [INSPIRE].

[37] M. Geracie, M. Goykhman and D.T. Son, Dense Chern-Simons Matter with Fermions at Large- $N$, arXiv: 1511.04772 [INSPIRE].

[38] S.H. Shenker and X. Yin, Vector Models in the Singlet Sector at Finite Temperature, arXiv: 1109.3519 [INSPIRE].

[39] T.T. Wu and C.N. Yang, Dirac Monopole Without Strings: Monopole Harmonics, Nucl. Phys. B 107 (1976) 365 [inSPIRE].

[40] O. Aharony, P. Narayan and T. Sharma, On monopole operators in supersymmetric Chern-Simons-matter theories, JHEP 05 (2015) 117 [arXiv: 1502.00945] [INSPIRE].

[41] C. Romelsberger, Counting chiral primaries in $N=1, D=4$ superconformal field theories, Nucl. Phys. B 747 (2006) 329 [hep-th/0510060] [INSPIRE].

[42] J. Kinney, J.M. Maldacena, S. Minwalla and S. Raju, An Index for 4 dimensional super conformal theories, Commun. Math. Phys. 275 (2007) 209 [hep-th/0510251] [INSPIRE].

[43] J. Bhattacharya, S. Bhattacharyya, S. Minwalla and S. Raju, Indices for Superconformal Field Theories in 3, 5 and 6 Dimensions, JHEP 02 (2008) 064 [arXiv:0801.1435] [INSPIRE].

[44] E. Witten, $\operatorname{SL}(2, \mathbb{Z})$ action on three-dimensional conformal field theories with Abelian symmetry, hep-th/0307041 [INSPIRE].

[45] S. Giombi and X. Yin, The Higher Spin/Vector Model Duality, J. Phys. A 46 (2013) 214003 [arXiv:1208.4036] [INSPIRE]. 